\title{
La responsabilidad de la universidad en la transformación de la matriz productiva del sector automotriz del Ecuador
}

\section{The responsibility of the university in the productive matrix transformation of the automotive sector of Ecuador}

\author{
Diana Belén Peralta Zurita \\ Universidad Internacional SEK Ecuador, Ecuador \\ Jaime Vinicio Molina Osejos \\ Universidad Internacional SEK Ecuador, Ecuador
}

Autor para correspondencia: diana.peralta@uisek.edu.ec

Fecha de recepción: 14 de Noviembre de 2017 - Fecha de aceptación: 15 de Mayo de 2018

Resumen: En los últimos años se ha comprendido que el sector automotriz requiere un alto aporte en diseño, procesos de manufactura y ensamble, detectándola como un área de oportunidad para el desarrollo, es por esto que las instituciones de Educación Superior ya no pueden ser entes de cátedra puramente locales, especialmente los centros de formación técnica universitaria, es inseparable de su capacidad para interactuar en procesos intelectuales, diálogos científicos ampliados, desarrollo e innovación; incorporando a sus funciones de enseñanza: la investigación, el estudio, análisis y búsqueda de nuevas áreas de oportunidad para la mejora, desarrollo, manufactura de productos y servicios que sean deseables, factibles y viables para el desarrollo de la sociedad e industria ecuatoriana. Con el compromiso ferviente des ser parte de esta evolución e innovación, la universidad tiene la gran responsabilidad de vincular y a ser partícipes a sus estudiantes y docentes, en proyectos reales de gran impacto, donde la industria ecuatoriana sea la beneficiada con este aporte.

Palabras Claves: industria ecuatoriana; matriz productiva; desarrollo; manufactura; productos

\begin{abstract}
In the last few years it has been understood that the automotive sector requires a high contribution in design, manufacturing processes and assembly, detecting it as an area of opportunity for development for that reason the higher education institutions cannot be purely academic, nowadays these training centers especially, technical education, are inseparable from the ability to interact on intellectual processes, scientific dialogues, development and innovation; Which includes teaching functions as: research, study, analysis and search of new areas of improvement opportunity, development, products manufacture. And desirables, feasible and viable services and products for the development of Ecuadorian society and industry.

With passionate commitment to contribute in this evolution and innovation, the university has the great responsibility of linking and being participants to its students and teachers, in real projects with great impact, where is benefited the Ecuadorian industry by this contribution.
\end{abstract}

Key Words: ecuadorian industry; products manufacture; development; ecuadorian products 


\section{Introducción}

El mundo cada vez es más complejo y difícil de interpretar. Múltiples esfuerzos tecnológicos, controlados y competitivos actúan en un determinado contexto para dar forma a las reglas de lo que es posible y probable. El descubrimiento de las más valiosas oportunidades innovadoras es un reto cada vez más complicado de lograr, especialmente aquellos que utilizan las herramientas tradicionales.

Los desafíos de todo el claustro universitario deben ser orientados a ser partícipe en la transformación de la matriz productiva iniciando con la incentivación de educandos y educadores a ser aporte con ideas innovadoras y retadoras que permitan la interrelación de fronteras científico-técnica, produciendo así una conversión en el sistema tradicional.

Para lograr este progreso es fundamental el incremento de las capacidades intelectuales y competencias de los profesionales donde el enfoque de la educación superior se encuentre centrado en el estudiante y su capacidad de aprender, exigiendo así más protagonismo y un alto compromiso con la sociedad y con el medio industrial. (Bautista \& Borges, 2006)

Sin dudar aún, la necesidad que se tiene del medio, al fácil acceso de nuevas tecnologías y la adquisición de herramientas, que permitan la generación de nuevas estrategias para mejora; desarrollando así productos y servicios que consoliden: competitividad, innovación, calidad, sostenibilidad, sustentabilidad y responsabilidad social.

\section{Componente teórico}

Cuando se inicia la búsqueda de la innovación, la mayoría de la gente asume equivocadamente que simplemente se está buscando generar una gran idea. La necesidad por parte de las empresas e industria ecuatoriana, en ofrecer productos y servicios que aporten a sus clientes un valor agregado, cada vez es mayor, buscando que esto sea a un precio más competitivo.

En la mayoría de los casos, la innovación se refiere como la introducción de la nueva tecnología en un producto o en su proceso de fabricación con el fin de mejorar su rendimiento y facilidad de uso o para minimizar su costo.

En el objetivo 10 del Plan nacional del buen vivir ecuatoriano (2013-2017) se menciona que:

"Los desafios actuales deben orientar la conformación de nuevas industrias y la promoción de nuevos sectores con alta productividad, competitivos, sostenibles, sustentables y diversos, con visión territorial y de inclusión económica, (...) promover la sustitución de importaciones, desagregación y transferencia tecnológica, conocimiento endógeno, y priorizar la producción nacional diversificada, con visión de largo plazo en el contexto internacional. " (Secretaría Nacional de Planificación y Desarrollo, 2013-2017, pág. 292)

Por esta razón es importante encontrar modernas y nuevas alternativas para la mejora y transformación de ciertos sectores estratégicos de relevancia industrial dentro del Ecuador, 
basado en esto se realiza un análisis de los ejes de cambio mencionados en el plan de la secretaria de Planificación y Desarrollo donde se nombra a 14 sectores productivos y 5 industrias prioritarias, para el proceso de cambio de la matriz productiva del Ecuador, como se muestra en la Tabla1:

Tabla 1. Industrias priorizadas del Ecuador

\begin{tabular}{ll}
\hline Sector & Industrias \\
\hline Bienes & 1) Alimentos frescos y procesados \\
& 2) Biotecnología (bioquímica y biomedicina) \\
& 3) Confecciones y calzado \\
& 4) Energías renovables \\
& 5) Industria farmacéutica \\
& 6) Metalmecánica \\
& 7) Petroquímica \\
8) Productos forestales de madera \\
9) Servicios ambientales \\
10) Tecnología (software, hardware y servicios informáticos) \\
11) Vehículos, automotores, carrocerías y partes \\
12) Construcción \\
13) Transporte y logística \\
14) Turismo
\end{tabular}

Nota: Los datos son tomados del estudio realizado por la Secretaria de Planificación y Desarrollo SENPLADES, $p$ 15. Derechos reservados (C SENPLADES [2012]

Al haber definido estos sectores de prioridad, se toma la decisión a partir del año 2012 de implementar políticas tributarias como medidas que promuevan la redistribución, fomento al empleo, la producción de bienes y servicios, adicionando a esto la responsabilidad ecológica y económica, incrementando ciertos impuestos a bienes y servicios como: importaciones dependiendo de la cantidad de artículos, materias primas, productos, partes, entre otros.

Anteriormente este tipo de elementos no grababan tributaciones, en caso particular las autopartes (CKD) requerimientos del sector automotriz, siendo así que "Los vehículos ensamblados en Ecuador contienen entre un 15\% y 22\% de componente local, según la CINAE" (El sector automotor, 2016). Por otro lado, el Ministerio de Industrias reporta que, del total de los automotores y componentes ensamblados dentro del país, únicamente el $7 \%$ es de fabricación nacional.

Aquí se evidencia que a pesar de que las estadísticas varían, la producción es sumamente baja, tanto en la fabricación de piezas automotrices como el producto ya terminado. "La industria automotriz produce 6 tipos de vehículos y 11 distintos productos de autopartes" (Alvarez, 2015). Con respecto a las causas de la baja productividad nacional de autopartes, Navarrete y Santamaría indican:

Según el ingeniero Baltazar Díaz inversionista de la empresa autopartes Andina S.A. ... la industria autopartista local enfrenta un grave problema en la actualidad, esto se debe a la enorme competencia que generan las importaciones de autopartes y repuestos procedentes de China, muchas 
empresas nacionales se han visto en la obligación de cerrar porque no se puede competir con los precios del país asiático, pero todo inicia porque no se pueden procesar la materia prima a nivel nacional (2016, p.32).

Obedeciendo a una política económica y social con un énfasis en la equidad, la progresividad fiscal y la redistribución de la riqueza. (Servicio de Rentas Internas del Ecuador, 2012). Y al haber una desventaja competitiva entre el sector productor nacional y las empresas importadoras, el gobierno impuso aranceles como medida para reducir el número de vehículos, piezas y autopartes importadas, incentivando el comercio de los productos nacionales. Debido a esto "desde el 2012 se exige a las ensambladoras tener el 5\% de contenido de Material Originario Ecuatoriano (MOE)"

Actualmente, el sector automotriz tiene una implicación importante en la economía del país producto de los ingresos generados en actividades económicas directas e indirectas, donde involucran otros sectores de prioridad de la industria como como la siderúrgica, metalúrgica, metalmecánica, minera, petrolera, petroquímica, del plástico, vidrio, electricidad, robótica e informática, industrias esenciales para la elaboración, fabricación y ensamblaje de vehículos.

Teniendo aquí a los grupos de autopartistas, proveedoras de partes y piezas quienes dependen de los estándares productivos de las cadenas, firmas y marcas de automotores de las cuales en el país se encuentran cuatro empresas que ensamblan vehículos de las marcas Chevrolet, Mazda, Kia y Hyundai y alrededor de 92 empresas productoras de autopartes, 1271 comercializadores e importadoras, 81 empresas carroceras y 3126 establecimientos de comercio automotor, quienes hasta el 17 de Noviembre del 2106 han generado 56801 empleos. (AEADE Asociación de Empresas Automotrices del Ecuador, 2017)

Ejemplo de algunas de las empresas más representativas encargadas de la producción nacional es Metaltronic S.A. que produce ciertos repuestos y piezas para los CDK (Díaz \& Tufiño, 2016). Tecnifaissa S.A. al igual que Autopartes Andina S.A. se ocupa de la producción de filtros principalmente, tanto para gasolina, lubricantes y aire. Productores como Tecnifaissa S.A. poseen convenios con marcas como Kia y Toyota, para las cuales realizan piezas bajo pedido, aunque las pruebas de calidad se realizan por muestreo (Navarrete \& Santamaría, 2016, p. 23). Por otro lado, Imfrisa S.A. es parte de un grupo colombiano que opera en el Ecuador fabricando, embragues, frenos, suspensiones y desde 2011 es el productor de repuestos originales de la marca Chevrolet (CINAE, 2017)

Si se realiza un análisis de las industrias que han sido grandes demandantes de tecnología y potencial humano, que a pesar de haber generado la cantidad mencionada de empleos, se ha visto afectada por el porcentaje de autopartes que aún no se generan en el país y que tiene que ser importadas pagando altos aranceles, debido a que no existe aún la materia prima necesaria, la tecnología y herramentales para la manufactura o por la falta de capital humano con los conocimientos necesarios para generar innovación.

En la figura 1 se puede observar que la producción de vehículos del mes de mayo 2017 ha sido de 3.043 unidades, mientras que en el año 2015 la producción fue de 3.849, reconociendo que aún no se logra superar la producción mensual desde hace 2 años en el medio. 


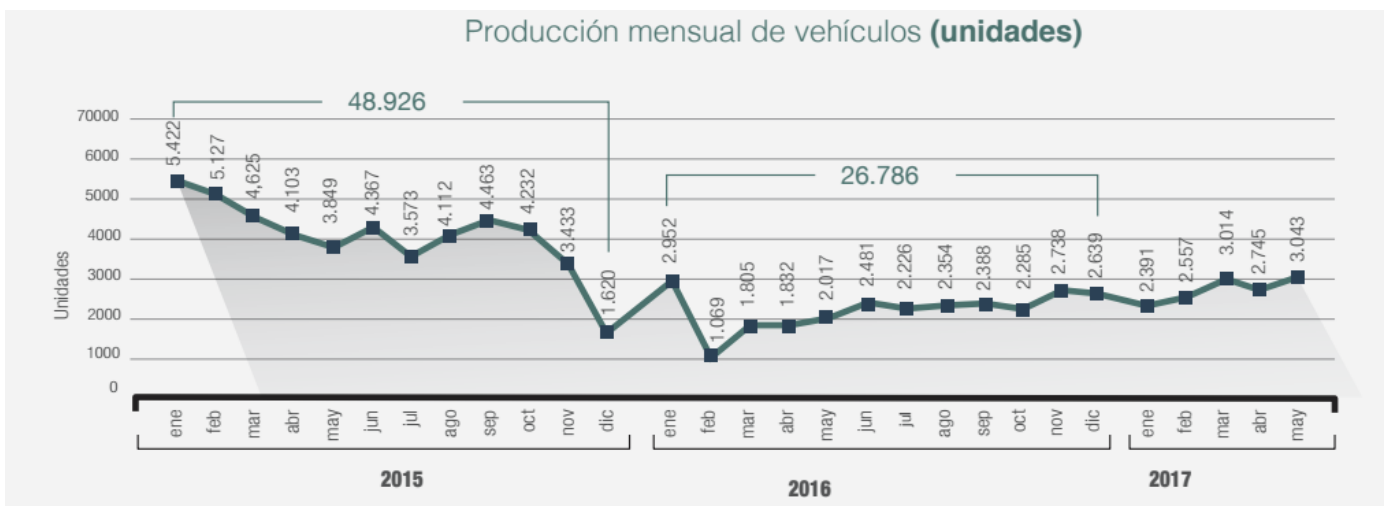

Figura 1. Producción mensual de vehículos Ecuador

Fuente: Cámara de la Industria Automotriz Ecuatoriana (CINAE), 2017.

Mientras que las cifras muestran la variación entre junio del año anterior y del actual un incremento del $11 \%$ en ventas de vehículos livianos, de la misma manera a nivel regional en el mes de junio, Ecuador y Argentina muestran crecimientos en las ventas de 111,4\% y 39,8\% respectivamente; mientras que los líderes en Latinoamérica del sector automotriz como: Venezuela y México registraron los mayores descensos en ventas (20,2\% y 5,2\%). Siendo la marca liderante del mercado ecuatoriano Chevrolet con un $42 \%$ de participación con los vehículos livianos, tipo camioneta y SUV como se muestra en la Figura 2:

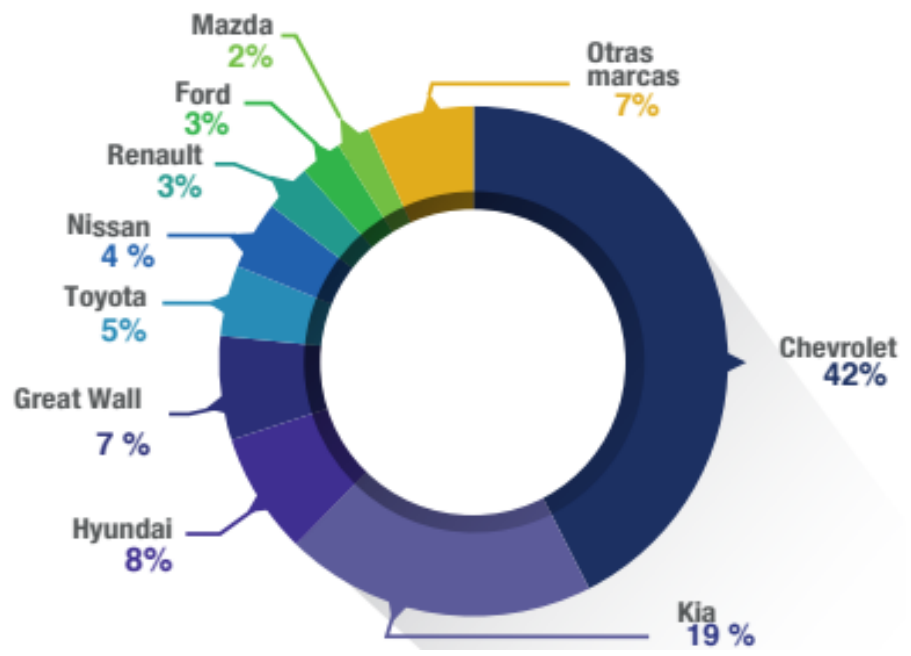

Figura 2. Participación de ventas por marcas 2017

Fuente: Asociación de Empresas Automotrices del Ecuador (AEADE), 2017 Autoplus, 2017

A pesar de todos los esfuerzos realizados desde el 2012 hasta el momento, no se logra superar las cifras de producción y exportación de vehículos manufacturados en el país, en la Figura 3, se puede observar el histórico del comportamiento en fabricación y salida de vehículos e interés por parte de otros países al mes de mayo desde el 2012 al 2017 : 


\section{PRODUCCIÓN Y EXPORTACIÓN DE VEHÍCULOS}

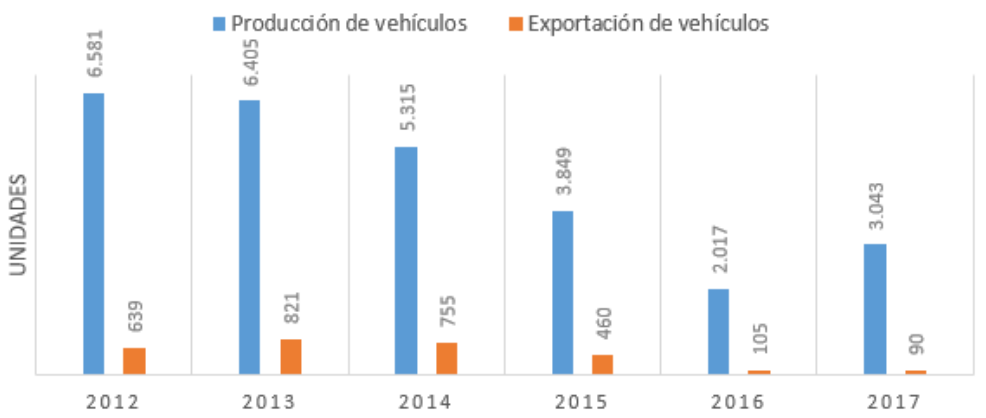

Figura 3. Producción y exportación de vehículos

Fuente: Cámara de la Industria Automotriz Ecuatoriana (CINAE), 2017.

Después de varias políticas públicas y tributarias que se han generado durante estos últimos 5 años y después de realizar un breve análisis de los resultados obtenidos en el sector automotriz, se propone una posible solución como hipótesis al aporte de la industria ecuatoriana.

Como posible alternativa de superación al subdesarrollo en el sector productivo del Ecuador se requiere incorporar alianzas estratégicas trianguladas como la metodología teórica presente en el triángulo de Sábato, donde se muestra tres ejes fundamentales de la sociedad como lo son: gobierno, infraestructura científica - tecnológica (Universidad) y estructura productiva (gobierno e industria), mencionadas en la Figura 4, las mismas que deben entender cuáles son las áreas de oportunidad en el medio y que puede ser significativo y de interés para cada uno de los ejes.

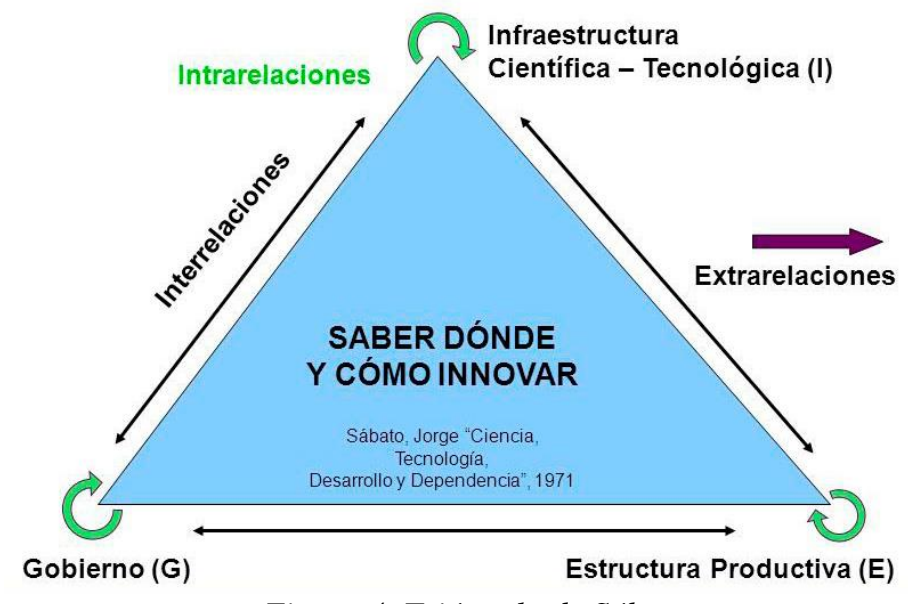

Figura 4. Triángulo de Sábato

Fuente: Sábato, Jorge "Ciencia, tecnología, desarrollo y dependencia”

Establecer este tipo de estrategia e incorporando la evolución en las relaciones entre universidades, empresas y gobierno, permite asumir nuevas tareas en el desarrollo de la tecnología de un país (Cardoso, 2006), estableciendo los roles de estos agentes de cambio de la siguiente manera: 
Soporte e intervención por parte de las universidades y centros de educación superior del país, no únicamente en capacitación, sino, en transferencia de conocimientos y tecnología a través de su alumnado y cuerpo docente, realizando procesos de vinculación para desarrollar proyectos de innovación dentro de las empresas. En este contexto, se requiere presentar esfuerzos conjuntos entre diversas instancias a favor de estrechar y fructificar la relación entre la educación superior y el sector productivo, asumiendo por parte de las instituciones educativas la oportunidad de desarrollo a la que pueden acceder según sus propias características y orientaciones institucionales.

Adicional a esto, como una alternativa para el cambio e innovación de la estructura productiva, es importante la interacción del estado como un agente ejecutor de políticas para el desarrollo económico-industrial. Así mismo, que facilite la interacción de las universidades con la industria.

Este triángulo de Sábato, muestra el modelo de política científico-técnico que años atrás han puesto en marcha otros países como lo muestra Aida Alvarado-Borrego en su trabajo "Vinculación Universidad - Empresa Y Su Contribución Al Desarrollo Regional" realizado en México, donde se presenta el análisis y reflexión de la importancia en el desarrollo de un país al dar impulso a la relación de vinculación Universidad - Empresa, de tal forma que su reciprocidad coadyuve en el fortalecimiento del desarrollo regional, impactando mediblemente en la sociedad, en sus procesos de generación de empleo, en la transferencia de conocimiento, de tal manera que impulse la competitividad de las instituciones de educación superior y de las empresas. (Borrego, 2009)

Otro ejemplo, en Argentina las políticas de promoción de distritos industriales y asociatividad surgieron como parte de estrategias nacionales de sustitución de importaciones, donde los gobiernos nacionales, provinciales y locales insertaron los conceptos de asociatividad, clusters y agrupamiento empresarial a través de programas que impulsaban el desarrollo del tejido productivo de las regiones favoreciendo la competitividad, la innovación, la gestión del conocimiento y el desarrollo regional apoyada por una línea política a las Pymes teniendo como expectativa la estimulación del aprendizaje y la acción colectiva entre empresas, con la generación, difusión y utilización del conocimiento como una actividad clave dinamizadora de la competitividad sistémica. (Mónica de Arteche, 2013)

Es por esto que se propone a los centros de educación y formación superior en ser partícipes y eje fundamental en el desarrollo del sector automotriz, existiendo áreas de oportunidad en el diseño de autopartes, diseño de vehículos motorizados, manufactura, generación y producción de nuevos materiales compuestos y biodegradables, es decir un amplio campo para vinculación de los profesionales y estudiantes en particular de las siguientes ramas de la ingeniería: mecánica, automotriz, diseño industrial, control de procesos, manufactura, industrial, mecatrónica, electrónica, digital, electromecánica, etc.

Si se observa en la Figura 5. El porcentaje de conformación de la industria y sector automotriz, donde la universidad por medio de su saber aporta: al desarrollo tecnológico, a la generación de proyectos e innovación. La misma que está conformada por 4 ensambladoras, 92 firmas autopartistas, 1408 comercializadoras e importadoras, 81 empresas carroceras, 3126 otras 
dedicadas a diversas actividades de servicio y comercio automotor. (AEADE Asociación de Empresas Automotrices del Ecuador, 2017)

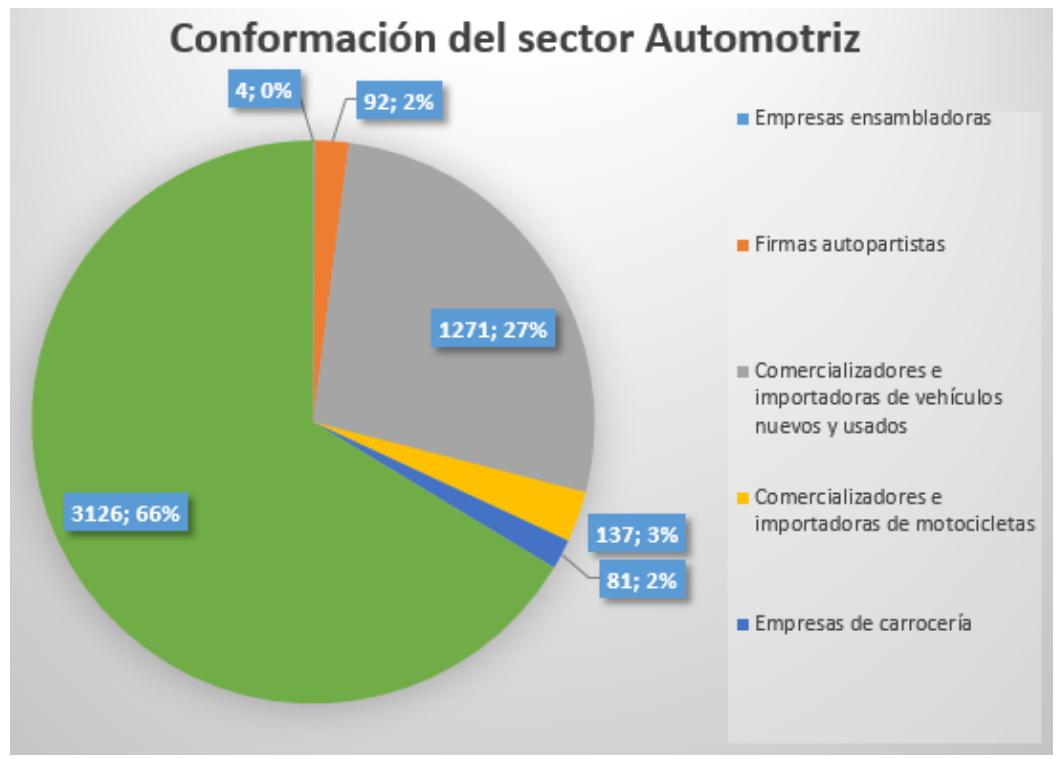

Figura 5. Conformación del sector automotriz 2017

Fuente: Asociación de Empresas Automotrices del Ecuador.

Considerando la oferta académica de educación superior de tercer nivel en el país que puede ser aporte al sector automotriz se consideran las siguientes instituciones para este nivel de formación en la Figura 6:

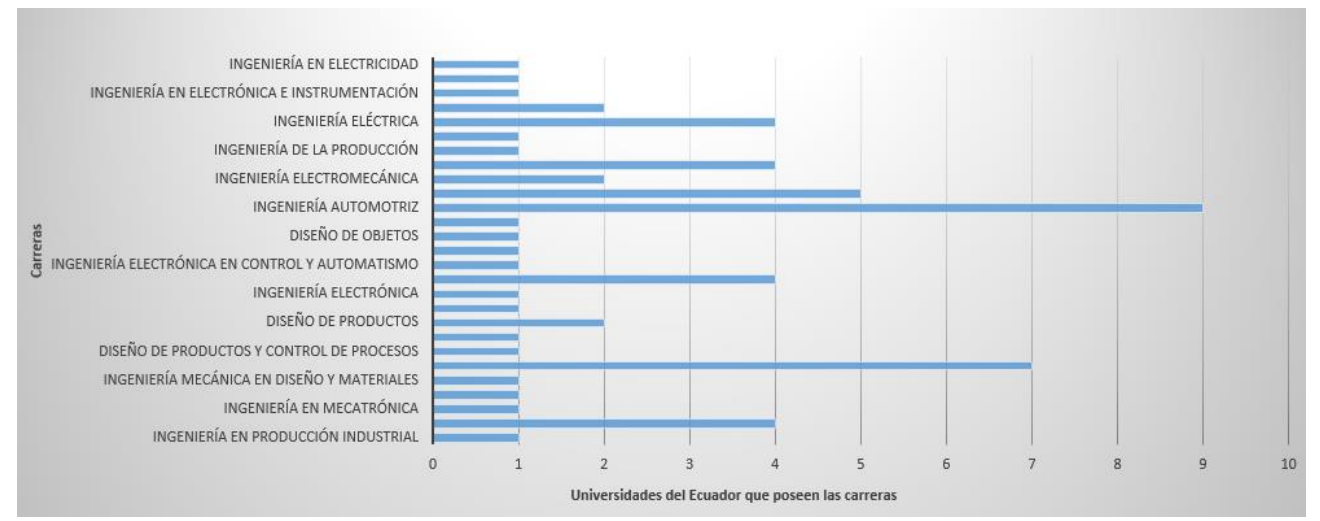

Figura 6. Instituciones con carreras de interés para el sector automotriz Fuente: Asociación de Empresas Automotrices del Ecuador.

Sin embargo, únicamente se cuenta con cuatro maestrías profesionalizantes como es: La Maestría en Diseño Mecánico con mención en autopartes de la Universidad Internacional SEK del Ecuador, la Maestría en Diseño y Simulación de la Escuela Politécnica Nacional, la Maestría en Mecánica de la Universidad Técnica de Ambato y la Maestría en Diseño Mecánico de la Escuela Politécnica de Chimborazo. (La Secretaría de Educación Superior, 2017) 
Es decir, se puede contar con profesionales que se encuentren en los últimos niveles de las carreas de pregrado y de posgrado, o a su vez con alumnos recién egresados y graduados de las anteriores mencionadas carreras de interés.

Según datos de la Senescyt, entre el 2010 y el 2015 el número de titulados, según el campo de conocimientos de ingeniería, industria y construcción, fueron un total de 39.462 personas entre hombres y mujeres con estudios de tercer nivel donde el $24 \%$ se titularon en el 2015; mientras que los de cuarto nivel en la misma área de conocimiento fueron de 2.140, siendo el 43\% de este valor titulados en el 2015, (La Secretaría de Educación Superior, 2017). Si se considera dentro del área y sub áreas de conocimientos según la UNESCO, las de interés de los titulados mencionados serían las siguientes:

- Ingeniería y profesiones afines: Dibujo técnico, mecánica, metalistería, electricidad, electrónica, telecomunicaciones, ingeniería energética y química, mantenimiento de vehículos, topografía. (CES)

- Industria y producción: Alimentación y bebidas, textiles, confección, calzado, cuero, materiales (madera, papel, plástico, vidrio, etc.), minería e industrias extractivas. (CES)

\section{Debate y discusión}

Considerando los datos previamente expuestos del sector automotriz y evidenciando el talento humano que tanto a nivel de carrera como de maestría posee el país, se considera que la universidad debe empezar a ser mediadora entre la industria y el gobierno, generando convenios donde los alumnos más destacados puedan ser partícipes en proyectos de diseño, desarrollo e innovación.

Siendo fundamental la apertura de la empresa, bajo un acuerdo de mutua cooperación, se vería beneficiada económicamente, debido a que contará con mano de obra altamente capacitada en haberes y saberes que requiere la empresa con: alta creatividad, pensamiento crítico, que permita llegar a una innovación y aquí la universidad se vería beneficiada ya que su alumnado y docentes pueden ejercer y tener acceso a herramientas a nivel industrial donde pueden aplicar sus conocimientos y habilidades, llegando a un nivel mayor de competitividad profesional.

Es imprescindible que el gobierno sea parte de este convenio triangulado, mediante políticas fiscales que motiven a la empresa a ser partícipe activo, una posible alternativa es el establecimiento de políticas tributarías preferenciales, algún tipo de beneficio o descuento en el pago de impuestos empresariales, así las empresas optarían por abrir las puertas a los alumnos y si cumplen con diferentes cantidades de alumnos de carreras de pregrado y posgrado o políticas de cuotas, donde se involucra el desarrollo técnico y tecnológico.

Así, las empresas tendrían gente capacitada desarrollando proyectos reales y que muchas de las veces no se pueden pagar, o no se tiene el tiempo disponible, pero si la infraestructura; proyectos orientados al diseño, innovación, automatización y mejora continua. Claro ejemplo es México, el cuál es un país con una fuerte capacidad manufacturera e industrial, utilizando esta metodología, que rompe el celo y miedo en las empresas de que las mentes jóvenes propongan 
soluciones y cambios en la industria, siendo guiadas por profesionales con más experiencia, en particular el caso del Instituto Tecnológico de Estudios Superiores de Monterrey. Quien cada semestre lanza una convocatoria a las empresas para que presenten los proyectos donde los alumnos pueden realizarlos. Con este incentivo tributario se puede lograr un desarrollo más acelerado que el que actualmente vivimos.

\section{Bibliografía}

Bautista, G., \& Borges, F. y. (2006). Didáctica Universitaria en entornos virtuales de enseñanza - aprendizaje. Madrid: Ediciones Narcea.

Secretaría Nacional de Planificación y Desarrollo. (2013-2017). Plan Nacional del Buen vivir. Quito: El telegrafo. doi:ISBN 978-9942-07-448-5

CINAE. (2017). Anuario de la Industria Automotriz ecuatoriana 2017. Quito.

Servicio de Rentas Internas del Ecuador. (2012). Una Nueva Política Fiscal para el Buen vivir. La equidad como soporte del pacto fiscal. Quito. doi:ISBN: 978-9942-07-275-7

AEADE Asociación de Empresas Automotrices del Ecuador. (2017). Perfil del sector automotor del ecuador. Quito. Recuperado el 18 de 04 de 2017, de http://www.aeade.net/perfil-delsector-automotor-del-ecuador/

Cardoso, J. L. (2006). Nuevas políticas de ciencia y tecnología. Buenos Aires: CLACSO. doi: ISBN: 978-987-1183-62-3

Borrego, A. A. (2009). Vinculación Universidad - Empresa Y Su Contribución Al. Ra Ximhai, 407 -414. doi:ISSN: 1665-0441

Mónica de Arteche, M. S. (2013). Redes y clusters para la innovación y la transferencia del conocimiento. Impacto en el crecimiento regional en Argentina. Estudios Gerenciales, 127-138.

La Secretaría de Educación Superior, C. T. (2017). Geoportal SNIESE . Obtenido de http://www.senescyt.gob.ec/visorgeografico/

CES, C. d. (s.f.). Manual del usuario SNIESE - Anexo 4: Areas Y Subáreas Del Conocimiento Unesco. Obtenido de http://www.puce.edu.ec/intranet/documentos/PISP/PISP-AreasSubareas-Conocimiento-UNESCO-Manual-SNIESE-SENESCYT.pdf 